\title{
Spousal involvement and CPAP adherence: a two-way street?
}

\author{
Angela McDowell
}

Received: 20 May 2010 / Accepted: 22 May 2010 /Published online: 17 June 2010

(C) Springer-Verlag 2010

In this issue of Sleep and Breathing, Kelly Glazer Baron and colleagues of the University of Utah, in their paper "Spousal Involvement in CPAP Adherence among Patients with Obstructive Sleep Apnea," investigate the role of wives' support or lack thereof in their husbands' continuous positive airway pressure (CPAP) adherence. Dr. Baron and colleagues previously reported that the role of the spouse can impact the afflicted partner, and while spousal pressure to seek treatment has been studied [1], this is the first study examining spousal involvement, such as support, control, and the context of the relationship, with regard to adherence to CPAP treatment.

In her recent review, Dr. Wendy Troxel [2] discusses the wealth of studies examining relationship quality and health, but rightly points out the minutiae of studies specific to sleep health and the role of the relationship. Dr. Baron and colleagues are providing much needed data in this area by examining the role of spousal involvement and CPAP adherence. The results of their study show that the perception of wives' support aided in personal adherence only when the disease severity was high. Accordingly, following nights of low adherence, collaboration with wives increased the next day, but the largest increases in collaboration were reported in patients with low levels of relationship conflict. This study indicates that spousal involvement is limited in its role in adherence to CPAP treatment, specifically modified by contextual variables such as the perceived quality of the relationship and disease severity; however, when spousal involvement does matter,

A. McDowell $(\triangle)$

Pulm., Crit. Care \& Sleep Medicine,

Case Western Reserve University,

10900 Euclid Ave,

Cleveland, OH 44106, USA

e-mail: alm126@case.edu the type of involvement also matters-with support from the spouse predicting adherence.

In spite of the utility of this study, there are several limitations to consider. First, not all of the participants were objectively measured with CPAP adherence cards. About half of the participants were scored by self-reports alone. Although the self-reports and objective reports correlated relatively well $(r=0.64)$, multiple problems surface: (1) Were there pretreatment differences between patients with CPAP machines with objective adherence cards and those without? (2) What factors does the small variability in the correlation of the methods of adherence reporting represent? What is the root of the misperception of patients with and without objective reports? Secondly, the participants of this study are rather homogenous: all males and mostly Caucasian middleaged men. Sex differences have been reported in relationship conflict and health behavior, and the prevalence of obstructive sleep apnea (OSA) in other races differs. The conclusions of this study are narrowly restricted to one sex and race, and therefore, it is important to replicate these findings with objective adherence reports and to further investigate the roles of sex and race differences.

Future studies might also attack this worthy issue from a different angle by investigating the impact of living with a spouse with OSA on the spouse that does not have OSA. One of the driving factors for OSA patients to seek treatment is the snoring-induced sleep disturbance to the non-afflicted spouse. The extent that the spouse has insomnia-related sleep problems may contribute to the role of the relationship by creating resentment and anger. A longitudinal study would be ideal, but a more cost-effective cross-sectional study looking at changes in the role of the relationship at various relationship time points would also provide valuable information. For example, looking at couples who have began their relationships $<1$ year, from 
1 to 5 years, 5 to 15 years, or 15 or more years and correlating that with OSA symptom onset may provide insights about the role of OSA symptoms affecting the nonafflicted spouse. Is it possible that the role of relationship conflict was minimized due to studying a patient population with such a high mean number of years married? It would be interesting to know how couples who are closer to the beginning stages of their relationship handle new OSA symptom onset, CPAP use, and if there's more or less conflict. Are these relationships more likely to end and how does that affect adherence in the OSA patient?

In sum, research into the role of spousal involvement in CPAP adherence is an interesting area that has profound implications for the quality of life for both spouses that may reciprocally affect the other spouse and possibly in a timedependent manner. More quickly identifying sources of support and avenues of conflict may aid in the long-term adherence to CPAP use.

\section{References}

1. Baron KG, Smith TW, Czajkowski LA, Gunn HE, Jones CR (2009) Relationship quality and CPAP adherence in patients with obstructive sleep apnea. Behav Sleep Med 7:22-36

2. Troxel WM (2010) It's more than sex: exploring the dyadic nature of sleep and implications for health. Psychosom Med 72:1-9 